\title{
LIPOMATOUS PSEUDOHYPERTROPHY OF THE PANCREAS
}

\author{
BY \\ O. D. BERESFORD AND T. K. OWEN \\ From the Chest Clinic and Royal Victoria Hospital, Bournemouth
}

(RECEIVED FOR PUBLICATION APRIL 6, 1956)

Uniform enlargement of the pancreas, due to massive deposition of fat and associated with severe hypoplasia of the exocrine tissue, was described by Hantelmann (1931) as lipomatous pseudohypertrophy. It is a very rare condition, and only eight previously reported cases have been found in the literature. Seven of these were in infants or children and one in a man of 77 years.

The following two cases, both in young adults, are therefore recorded. The first case is of particular interest because of the multiplicity of the lesions ; dwarf stature, generalized scleroderma, rheumatic heart disease, chronic pulmonary tuberculosis, and a terminal haemorrhagic diathesis.

\section{Case Reports}

Case 1.-A woman, aged 27 years, was the youngest of nine children ( 6 males, 3 females). Two died in infancy from unknown causes. The rest of the family are well. She was first seen in February, 1953, because of pulmonary tuberculosis. Her main complaint at that time was of dyspnoea on exertion, but she stated that ever since she could remember her skin had been pigmented. In 1940 she had noticed that the skin of her fingers was becoming rather tight and some contracture of the fingers occurred at that time. In 1943 a diagnosis of rheumatic heart disease was made and she was admitted to hospital for several months. In 1949 she noted that the hair of the scalp was falling out. In 1950 she was admitted to hospital because of the skin condition. A chest radiograph at that time showed an enlarged heart and infiltration in the left upper and mid zones with cavitation. Sputum was positive for tubercle bacilli on direct smear. After approximately one year's treatment the tuberculosis was apparently stable and the patient was persistently sputum negative.

Clinical examination in February, 1953, showed a patient of small stature, height $59 \mathrm{in} .(150 \mathrm{~cm}$.) and weighing $88.5 \mathrm{lb}$. $(40.2 \mathrm{~kg}$.). There was a diffuse, dappled pigmentation of the skin of the face and trunk, and confluent pigmentation of the arms and legs. The skin over the hands and face was smooth and leathery. There was contracture of the inter- phalangeal and metacarpo-phalangeal joints of both hands. Callosities were present on all toes and on the balls of the feet, but there was no evidence of ulceration or scarring. The hair of the scalp was brittle and sparse, particularly over the vertex. The eyebrows were scanty and the axillary and pubic hair absent. The fundi oculi were normal and there was no evidence of cataract. The heart showed the clinical features of mitral stenosis and aortic incompetence, and the blood pressure was $110 / 60 \mathrm{~mm}$. Hg. A chest radiograph showed an enlarged heart and a fibrotic lesion at the left apex. Radiographs of the tibiae, fibulae, knee, and ankle joints showed no evidence of rarefaction.

Subsequently the patient remained fairly well until April 19, 1954, when she developed what appeared to be an influenzal attack, characterized by cough, general malaise, and fever. This was treated with sulphonamides, but on the following day she developed a severe epistaxis. The patient refused admission to hospital and remained at home under the care of her own doctor. Epistaxis continued and she developed upper abdominal pain. On April 27, 1954, her condition had deteriorated considerably and she was admitted to hospital, but died one and a half hours after admission before any investigations could be made.

Necropsy was performed 22 hours after death. The skin was of a mottled dark brown, thickened and lacking the usual elasticity. The hair was very scanty on the head and eyebrows, and there was no pubic and axillary hair. The trachea and bronchi contained much bright red blood. The right lung was very oedematous and the left lung showed fibro-caseous tuberculosis involving the whole of the upper lobe. There was a cavity approximately 1 in. $(2.5 \mathrm{~cm}$.) near the periphery of the lower part. The apex of the left lower lobe also showed some recent infiltration. The heart weighed $12 \mathrm{oz}$. (342 g.). The mitral valves showed typical rheumatic stenosis and the chordae tendinae were thickened and fused. The left auricle was somewhat dilated. The free edges of the cusps of the aortic valves were slightly rolled. Three small petechial haemorrhages were found on the surface of the heart. The liver weighed $28 \mathrm{oz}$. (798 g.) and was a tawny brown and appeared "fatty." The 
stomach contained a little altered blood, and the lower part of the small intestine and colon contained a large amount of tarry faeces. The spleen was small and weighed $4 \mathrm{oz}$. (114 g.). The kidneys were small, each weighing $3 \frac{1}{2} \mathrm{oz}$. (100 g.). There was haemorrhage into the pelvis of the left kidney. The brain showed a small subarachnoid haemorrhage around the right middle cerebral artery, spreading slightly on to the surface of the temporal lobe.

The pancreas was considerably enlarged, weighing 185 g. (normal, 90-120 g., Saphir, 1951). Its shape was normal, but the appearance and consistence was that of fatty tissue (Fig. 1). So much fat in the pancreas was in surprising contrast to its complete absence in the rest of the body. The cut surface showed a few white fibrous strands.

The immediate cause of death appeared to be multiple haemorrhages due to a haemorrhagic dia-

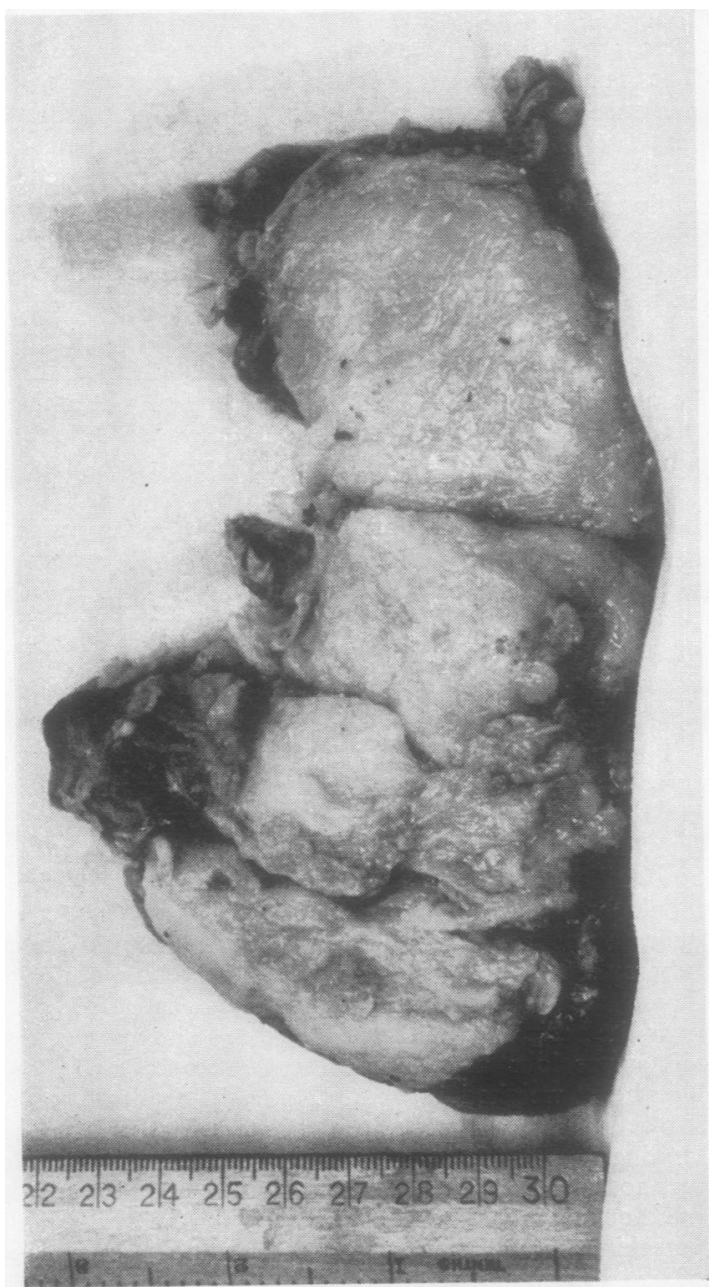

Fig. 1.-The pancreas of Case 1. The photograph was taken after three vertical incisions had been made.

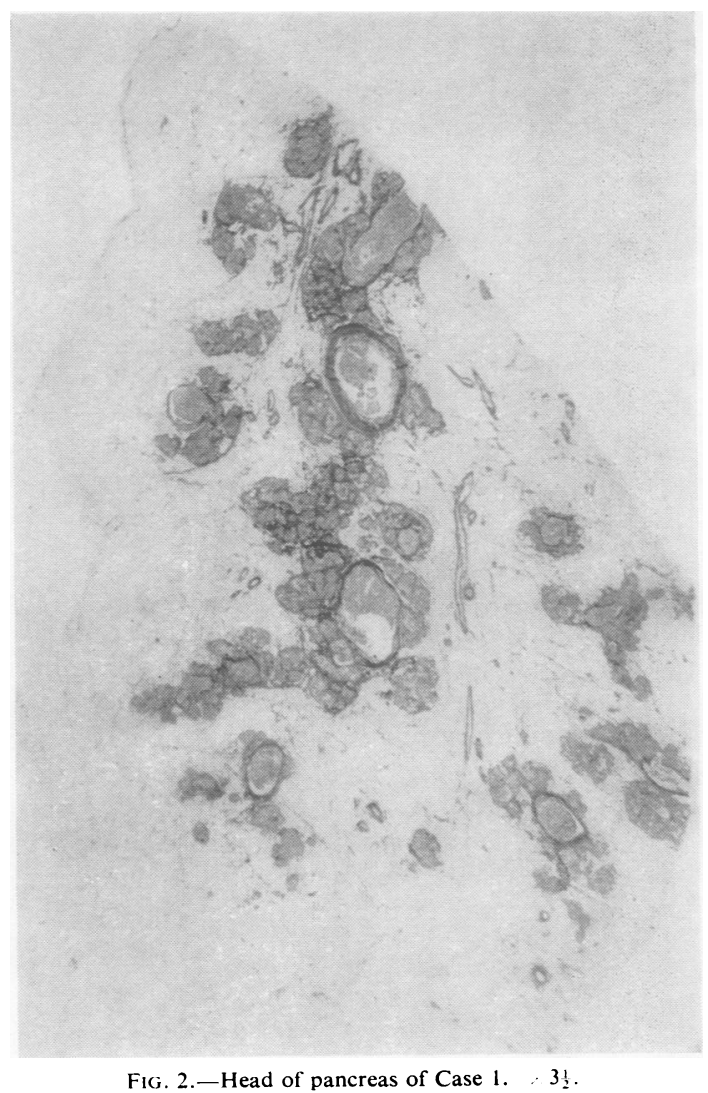

thesis, possibly caused by sulphonamides. This was associated with chronic pulmonary tuberculosis, rheumatic heart disease, and a skin lesion consistent with a diagnosis of scleroderma.

Histological Examination.-A section of the head of the pancreas is shown in Fig. 2. The fatty infiltration and the atrophy of the parenchyma can be clearly seen. Clusters of acini can be seen around the ducts. They are composed of cells with clear cytoplasm and showing no normal secretory granules (Fig. 3). The clusters are surrounded by fibrous tissue. Islets of Langerhans can be seen persisting in fatty tissue in areas from which all the exocrine elements have disappeared (Fig. 4). Sections of the body and the tail show a similar picture but with less surviving pancreatic tissues. The condition is clearly that described by Hantelmann (1931) as lipomatous pseudohypertrophy of the pancreas. Sections of the skin of the abdominal wall and scalp showed the characteristic changes of scleroderma, with atrophy of rete pegs. thickening of the collagen bands, very scanty hair follicles, and slight round-celled infiltration around the skin appendages. There was an excessive amount of pigment in the basal layers of the epidermis. Microscopically the liver showed very severe fatty degeneration. Sections of the left kidney showed 


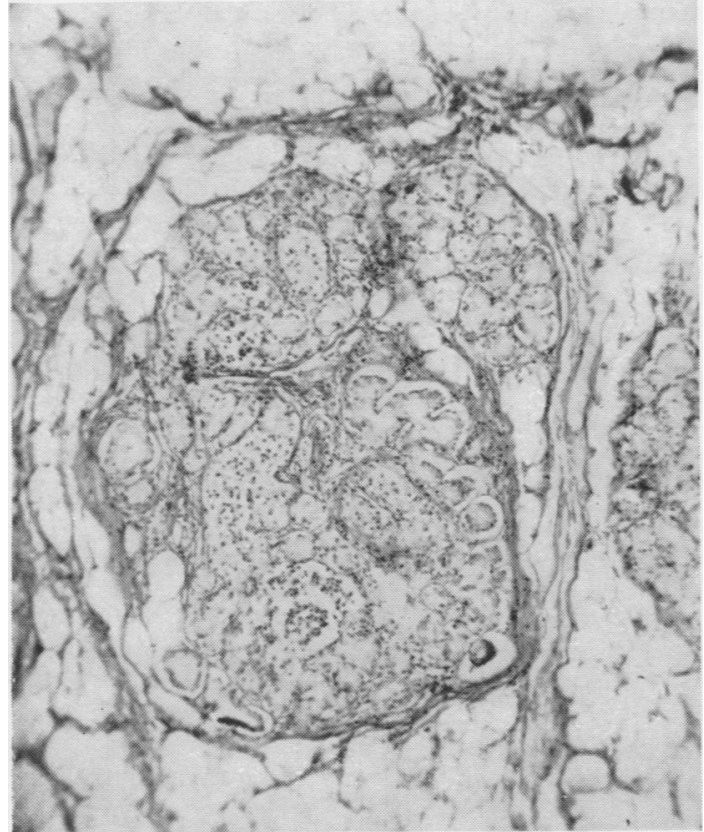

FIG. 3.-Pancreas of Case $1 . \times 60$.

much haemorrhage into the pelvis and some roundcelled infiltration around the glomeruli. Sections taken from other organs, including the pituitary, showed no significant changes.

Case 2.-A man, aged 23 years, was admitted to hospital on November 9, 1953. He had suffered from a troublesome cough for some few weeks before admission and had been becoming increasingly dyspnoeic for several days. On admission he complained of dyspnoea and feverishness. He stated that in childhood he had suffered from coeliac disease. Physical examination showed a dyspnoeic and somewhat cyanosed young adult. Respirations were 44 per minute, temperature $99.4^{\circ} \mathrm{F}$., and pulse 130 per minute. The chest was barrel-shaped with a poor expansion. A few crepitations were audible at the bases. The liver edge was palpable $2 \frac{1}{2}$ in. below the costal margin. Slight finger clubbing was noted. A blood count showed 11,000 leucocytes per c.mm. ( $80^{\circ}$ o polymorphs).

He was treated empirically with penicillin and streptomycin, but on November 14, 1953, his condition rapidly deteriorated and he died on that day.

Necropsy was performed 24 hours after death. The body was that of an emaciated young adult male. The trachea and bronchi contained a large amount of purulent material. The right middle lobe of the lung contained numerous small abscesses and the right lower lobe showed a confluent bronchopneumonia. The heart was normal. The liver was enlarged, weighing $70 \mathrm{oz}$. (1,995 g.) and markedly friable. The cut surface showed a yellow and white mottled appear-

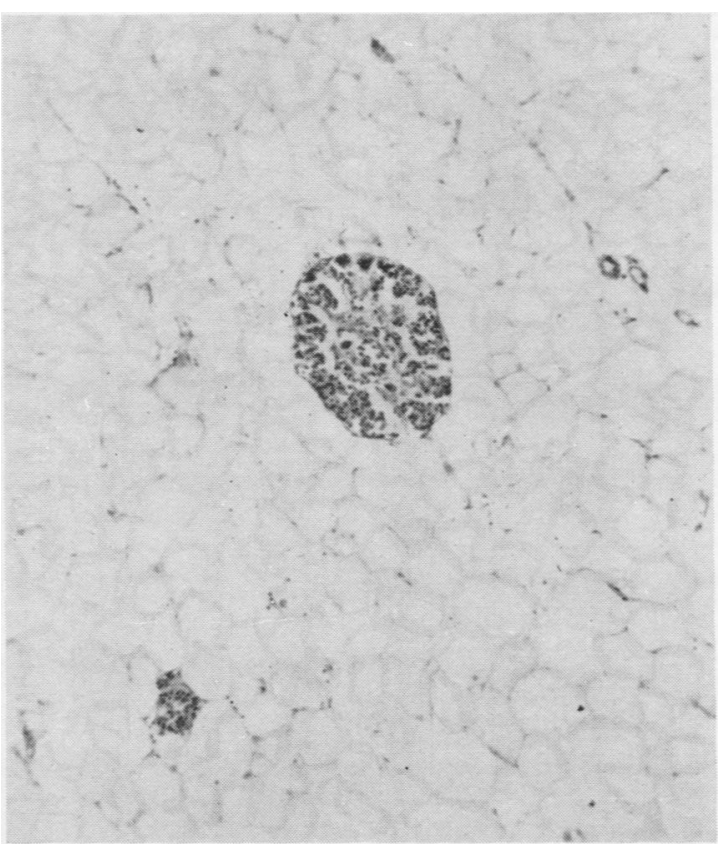

FIG. 4.-Persistent islet of Langerhans in fatty tissue in Case $1 . \times 60$.

ance. The pancreas was uniformly enlarged (330 g.) and white. On the cut surface no pancreatic tissue was visible, the whole having apparently been replaced by fat. The other organs showed no significant changes.

Microsopical examination of the pancreas showed the picture of fatty replacement of the acini with survival of the islets of Langerhans, as in Case 1. The liver showed severe fatty degeneration, roundcelled infiltration and fibrosis, and proliferation of the bile ducts in the portal area. The lung showed bronchopneumonia of a haemorrhagic type.

\section{Discussion}

Apart from the rarity, the interest of these two cases lies in the age incidence, both occurring in the third decade of life. The latest review of the subject is given by Robson and Scott (1953), who recorded a case in a man of 77 years. He suffered from diarrhoea and grossly impaired absorption of fat. In their review of previously reported cases they came to the conclusion that there is no constant pattern of clinical findings. Their review did not, however, include the cases reported by Davie (1938) and by Lumb and Beautyman (1952) under different names. Davie reported the condition in a mentally defective child aged 4 years, under the title "Massive Replacement of the Pancreas by Adipose Tissue." The size of the pancreas "was, if anything, larger than normal, and it pos- 
sessed the usual shape and superficial lobular markings." He considered "fat replacement" to be the most suitable term to use. Lumb and Beautyman reported two cases in siblings, aged 4 weeks and 23 months, under the title "Hypoplasia of the Exocrine Tissue of the Pancreas." They considered that to call the condition lipomatosis over-emphasized the purely replacement phenomenon, and that the primary lesion was the absence of exocrine tissue. In their second case the pancreatic changes were associated with only the beginnings of adipose tissue replacement. But in our two cases the term lipomatous pseudohypertrophy seems a very apt description, and quite valid in view of our lack of understanding of the real nature of the condition.

The aetiology is obscure. Høyer (1949) discussed the possibility of a congenital anomaly, in view of the incidence in childhood, but thought that the condition was more probably acquired, due to injury of the pancreatic parenchyma by infective or toxic agents. Another suggestion (Robson and Scott, 1953) is that it is a true lipoma. This would explain the persistence of fat while the rest of the body is wasted, which was very striking in our Case 1. Against this is the fact that the enlargement preserves the shape of the pancreas.

Case 1 presented an unusual complex of pathological lesions. At one time a diagnosis of Werner-Rothman syndrome (Thannhauser, 1945) was made. This is a hereditary-familial dermatosis associated with cataracts, premature baldness, callosities of the feet, ulceration of the shins, and $\overrightarrow{\vec{s}}$ endocrine features. However, in the absence of a family history and of cataracts, it seems that $\frac{C}{0}$ this diagnosis cannot be maintained. Histologi- $\overline{\bar{D}}$ cally the skin lesion was typical of scleroderma. The association of four distinct pathological lesions involving the skin, pancreas, lungs, and क heart appears to be fortuitous, but it may well be $\overrightarrow{0}$ that the pancreatic lesion made the patient more susceptible to the associated diseases.

\section{Summary}

Two cases of a rare disorder, lipomatous pseudohypertrophy of the pancreas, are described.

One case presented a multiplicity of pathological lesions, and it is suggested that the underlying lesion of the pancreas made the patient more $\vec{T}$ susceptible to scleroderma, rheumatic heart $\mathbb{D}$ disease, and pulmonary tuberculosis.

We wish to express our thanks to Dr. I. W. Whimster for his opinion on the section of the skin $\vec{f}$ from Case 1, and to Dr. J. H. Bentley for his clinical. notes of Case 2.

\section{REFERENCES}

Davie, T. B. (1938). J. Path. Bact., 46, 473.

Hantelman n, W. (1931). Virchow: Arch. path. Anat., 282, 630.

Hoyer, A. (1949). J. Path. Bact., 61, 93.

Lumb, G., and Beautyman, W. (1952). Ibid., 64, 679.

Robson, H. N., and Scott, G. B. D. (1953). Gastroenterology, 23, 74.

Saphir, O. (1951). Autopsy Diagnosis and Technic, 3rd ed. Heeber, 음 New York.

Thannhauser, S. J. (1945). Ann. intern. .Hed., 23, 559. 